Article

\title{
An Underactuated Bio-Inspired Helical Swimming Microrobot Using Fuzzy-PI Controller with Novel Error Detection Method for 5-DOF Micromanipulation
}

\author{
Mohammad Javad Pourmand ${ }^{1}$, Sajjad Taghvaei ${ }^{1}$, Ramin Vatankhah ${ }^{1}$ and \\ Mohammad Mehdi Arefi ${ }^{2, *}$ \\ 1 School of Mechanical Engineering, Shiraz University, Shiraz 71348-51154, Iran; \\ mpourmand@shirazu.ac.ir (M.J.P.); sj.taghvaei@shirazu.ac.ir (S.T.); rvatankhah@shirazu.ac.ir (R.V.) \\ 2 Department of Power and Control Engineering, School of Electrical and Computer Engineering, \\ Shiraz University, Shiraz 71348-51154, Iran \\ * Correspondence: arefi@shirazu.ac.ir; Tel.: +98-713-613-3073
}

Received: 8 April 2018; Accepted: 8 June 2018; Published: 14 June 2018

\begin{abstract}
The potential of microrobots to bring about revolutionary changes over micro-operation demands is increasing day to day. This paper presents a controller to provide 5 degrees of freedom for an underactuated bio-inspired helical swimming microrobot. The considered system is a helical swimming microrobot with three flagella in a low Reynolds performance environment. Control of the considered system is performed to reach any desired location, roll angle and pitch angle. The proposed controlling error definition extracted from the system geometry is general for similar actuation configurations. An error detection method for multi-propulsion-unit systems is utilized for 5-DOF micromanipulation of an underactuated bio-inspired helical swimming microrobot by fuzzy-PI controller. A fuzzy-PI controller is proposed to use modified experimental data of PI controller debugging to maintain a suitable efficient control. The comparison of two other possible controllers and the proposed fuzzy-PI controller is discussed, and the performance of trajectory tracking is evaluated by simulations.
\end{abstract}

Keywords: fuzzy-PI control; helical swimming microrobots; 5-DOF micromanipulation; system error detection

\section{Introduction}

Microrobots have various applications in industries. The most potent and also feasible field is biomedical application. Biomedical microrobots can be used for drug delivery [1], brachytherapy [2], stem cells implantation [3], artery ablation, biopsy, and so on [4]. The limitation of the microrobots maneuverability is always the most restrictive aspect of design and application of these instruments [4]. As far as the previous researchers show, the Reynolds number of the microdimensional vessels like venule, capillary, and small arteriole are low. Therefore a lot of studies in vascular applications used this fact for designing microrobots [4].

A large number of the microswimmers are designed inspired by bacterial flagella locomotion [4]. However, application of the moto-driven system in micro sizes is challenging and requires high-tech instrumentation. The best currently feasible option for the system propulsion is to rotate helix/helixes to make the bacterial flagella locomotion. The power transmission can be improved and the limited degrees of freedom could be resolved (anyhow by using nonlocal approaches) to prevent unnecessary costs such as size minimization of the locomotion system. Hence, fewer local instrumentations require innovative actuation systems using the powerful controllers. 
The methods proposed for manipulation in micro scales are as follows: magnetic resonance navigation (MRN) of spherical bead [5] and elliptical bead [6], magnetically driven elastic flagellated propulsion [7], magnetically driven rigid flagellated propulsion [8], electrostatic actuation [9], bubble-powered actuation [10], mechanical vibration actuation [11], photoactive polymer actuation [12], etc. The most dominant factor affecting the selection of the manipulation method is the application environment and the ultimate application environment of a great number of microrobots is a human body. In the human body not only is there the need for detection of all the resistive forces and uncertainties, optimal navigation in order to reduce resistive forces and uncertainties $[5,13]$, and robustness of control, but also the power needs to be transferred into the body. Therefore, the most effective manipulation method has been magnetic actuation [4]. Even though magnetic actuation shows good results in in vivo experimental validation with up to 5-DOF control, the under-operation body has size limitation [6].

To increase DOF, the simplest way is to enhance local instrumentations like the number of flagella and robot's joints [14]. In that case, the number of control signals will increase which leads to use of DC-motor [15], piezoelectric motor [16], ionic conducting polymer gel film (ICPF) actuation [17], artificial biomolecular motor [18], and so on, as the actuation method.

Since microrobots normally deal with systematic disturbances and structured uncertainties, so many robust methods of control have been proposed for their navigation such as predictive controller [19], $H_{\infty}$ controller [20] and adaptive controller [21]. However, there are different tools for controlling complex systems with uncertainties. Among these controllers, fuzzy logic controllers have shown good performance and efficiency. Fuzzy logic controllers are embedded a crucial characteristic called inference which acts similar to the human brain's decision-making and is literarily obtained from human judgments [22]. Fuzzy logic can be used in control systems which cannot be easily or accurately modeled [23] to eliminate the uncertainties of the system [24] or to encompass the nonlinearities of the system [25]. The most straightforward way to increase robustness of systems with determined systematic feedback is to use a fuzzy logic supervisor for coefficients of a simple proportional-integral-derivative (PID) controller [26]. In control systems, the Fuzzy-PI controller uses fuzzy logic algorithms to effectively eliminate the steady state error by adaptively adjusting proportional and integral coefficients of PI controller [27]. One of the significant approaches to control the nonlinear systems in the presence of model uncertainties is combining the fuzzy logic with gain scheduling and sliding mode technique. In [28], the model of the plant is modeled based on Takagi-Sugeno-Kang fuzzy approach and a constructive procedure is suggested to design a gain-scheduling controller. By partition of the state space into a finite number of fuzzy regions and benefiting from the idea of the sliding mode control scheme, a fuzzy gain-scheduler is proposed in [29]. Furthermore, the investigation of the stability and robustness of the gain scheduled closed loop control system is accomplished in [29]. The key point of the design of controllers using fuzzy logic is to detect and assimilate errors correctly and more efficiently.

In this paper, a flagellated bead with tree flagella was considered. The resistive forces against the propulsion of the microrobot in low Reynolds number were considered, feasible modeling assumptions were established, and the system's governing equations were elicited. A controlling error was driven from the system's geometry without further simplification or any further assumptions. Possible controllers were discussed, and a simple P-controller and a robust fuzzy-PI controller were considered. The simulations over the system's control were performed, and the purposed controller was evaluated. The proposed method can be implemented for other control systems such as underactuated robotic manipulators [30] and vibration control systems [31].

The rest of this paper is organized as follows: In Section 2, dynamic modeling of a 5-DOF microrobot is presented. Section 3 provides the control system and the proposed Fuzzy PI controller. Simulation results are presented in Section 4, and some concluding remarks are given in Section 5. 


\section{Dynamic Modelling}

To provide 5-DOF performance in micro scales and low Reynolds number fluid flows, a helical swimming microrobot with three propulsion flagella is considered. Because of the performance condition according to resistive-force, the inertial forces can be eliminated from governing equations. The flagella are located with the angular distance of $120^{\circ}$, distributed in and normal to YZ-plane as shown Figure 1. The robot body is a sphere of radius $\rho$.
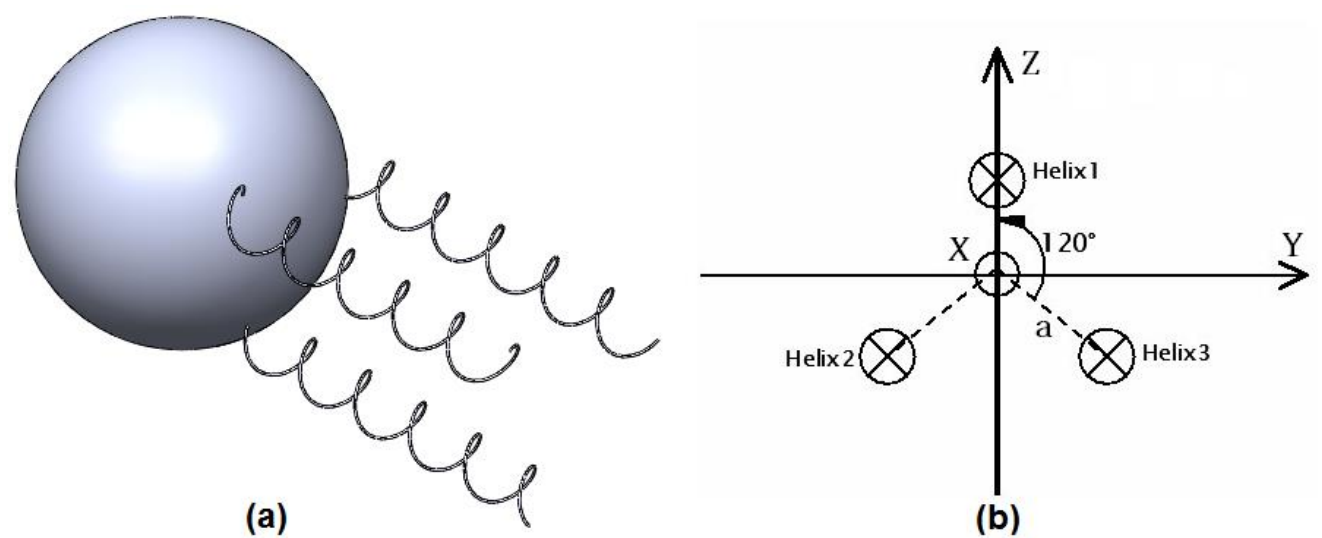

(a)

(b)

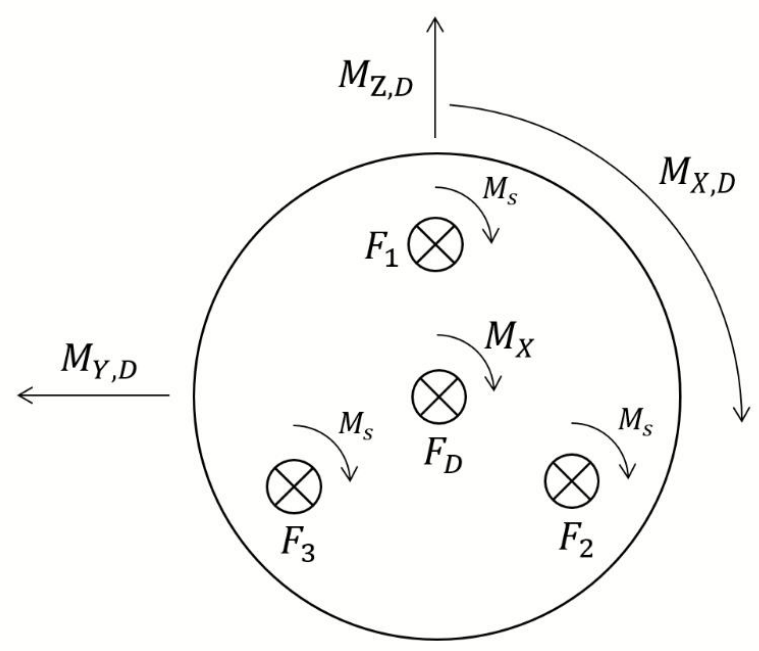

(c)

Figure 1. (a) The robot's schematic; (b) helixes position and orientation relative to the robot's body; (c) representation of forces and torques from $Y-Z$ plane.

The hydrodynamic of robot's body can be declared by a drag force and a drag torque due to its translation and rotation. The helixes produce viscous torque caused by their pure rotation about their axis and they are affected by the torque due to the body rotation.

There will be four static equilibriums governing the system as follows:

$$
\begin{gathered}
F_{D}+\sum_{i=1}^{3} F_{i}=0 \\
M_{X, D}+3 M_{s}+M_{X}=0 \\
M_{Y, D}+F_{1} a-\left(F_{2}+F_{3}\right) a / 2=0 \\
M_{Z, D}+\left(F_{2}-F_{3}\right) a \sqrt{3} / 2=0
\end{gathered}
$$


where $F_{D}$ is the drag force affecting the body, $M_{X, D}, M_{Y, D}$, and $M_{Z, D}$ are the drag torques affecting the body, $F_{1}, F_{2}$, and $F_{3}$ are the propulsion forces belonging to each flagella, $M_{s}$ denotes as viscous torque of the flagella due to the body rotation, and $M_{X}$ denotes the torque produced by the viscous interaction affecting the flagella. Considering Stokes flow for performance environment the drag force and the drag torques will be obtained as

$$
\begin{gathered}
F_{D}=6 \pi \mu \rho \dot{n} \\
M_{i, D}=8 \pi \mu \rho^{3} \dot{\Omega}_{i} \quad i=X, Y, Z
\end{gathered}
$$

Moreover, the viscous torque applied to each flagella is obtained as [32]

$$
M_{s}=N \pi \lambda \mu d^{2} \dot{\Omega}_{X} \cos \beta
$$

where $\mu$ is the fluid dynamical viscosity, $d$ is the filaments radial thickness, $\Omega$ is angular velocity about each axis, $\lambda$ is the helix wavelength, and $N \lambda$ is the total length of the helix. $\beta$ denotes the pitch angle of the helix and can be determined by

$$
\tan \beta=2 \pi r / \lambda
$$

where $r$ is the circular helix radius. The viscous forces of the flagellum have to be obtained to bring the governing equations to an application. On the element of length along the helix, the normal and tangential components of the viscous force element can be calculated as follows:

$$
\begin{gathered}
d F_{n}=-C_{n} V_{n} d s \\
d F_{s}=-C_{s} V_{s} d s
\end{gathered}
$$

where $V_{n}$ and $V_{s}$ are the normal and tangential velocity of the length element, $d s, C_{n}$ and $C_{s}$ are resistive coefficients computed from $\mu, \lambda$, and $d$ [32]. The velocities are obtained by

$$
\begin{gathered}
V_{n}=-\dot{X} \sin \beta+r\left(\omega-\dot{\Omega}_{X}\right) \cos \beta \\
V_{s}=\dot{X} \cos \beta+r\left(\omega-\dot{\Omega}_{X}\right) \sin \beta
\end{gathered}
$$

where $\omega$ is the angular velocity of each helix about $X$-axis. Also, the elements of the hydrodynamic torque and force along $X$-axis for each flagellum are

$$
\begin{gathered}
d F_{X}=d F_{s} \cos \beta-d F_{n} \sin \beta \\
d M_{X}=r\left(d F_{n} \cos \beta+d F_{s} \sin \beta\right)
\end{gathered}
$$

Total length of the flagellum along the helix is equal to $N \lambda \cos \beta$, so by substitution of Equations (9)-(12) into Equations (13) and (14) and integration, we have

$$
\begin{gathered}
F_{i}=r N \lambda\left(C_{n}-C_{S}\right)\left(\omega_{i}-\dot{\Omega}_{X}\right) \sin \beta-N \lambda\left(C_{n} \sin ^{2} \beta \sec \beta+C_{s} \cos \beta\right) \dot{X} \quad i=1,2,3 \\
M_{X}=r N \lambda\left(C_{n}-C_{s}\right) \dot{X} \sin \beta-r^{2} N \lambda\left(C_{s} \sin ^{2} \beta \sec \beta+C_{n} \cos \beta\right)\left(\sum_{i=1}^{3} \omega_{i}-3 \dot{\Omega}_{X}\right)
\end{gathered}
$$

Substituting Equations (5)-(7), (15) and (16) into Equations (1)-(4) yields

$$
-6 \pi \mu \rho \dot{X}+\sum_{i=1}^{3} r N \lambda\left(C_{n}-C_{s}\right)\left(\omega_{i}-\dot{\Omega}_{X}\right) \sin \beta-N \lambda\left(C_{n} \sin ^{2} \beta \sec \beta+C_{s} \cos \beta\right) \dot{X}=0
$$




$$
\begin{aligned}
& 8 \pi \mu \rho^{3} \dot{\Omega}_{X}+3 N \pi \lambda \mu d^{2} \dot{\Omega}_{X} \cos \beta+r N \lambda\left(C_{n}-C_{s}\right) \dot{X} \sin \beta-r^{2} N \lambda\left(C_{s} \sin ^{2} \beta \sec \beta+C_{n} \cos \beta\right) \\
& \left(\sum_{i=1}^{3} \omega_{i}-3 \dot{\Omega}_{X}\right)=0 \\
& 8 \pi \mu \rho^{3} \dot{\Omega}_{Y}+\left(r N \lambda\left(C_{n}-C_{s}\right)\left(\omega_{1}-\dot{\Omega}_{X}\right) \sin \beta-N \lambda\left(C_{n} \sin ^{2} \beta \sec \beta+C_{s} \cos \beta\right) \dot{X}\right) a- \\
& \left(\sum_{i=2}^{3} r N \lambda\left(C_{n}-C_{s}\right)\left(\omega_{i}-\dot{\Omega}_{X}\right) \sin \beta-N \lambda\left(C_{n} \sin ^{2} \beta \sec \beta+C_{s} \cos \beta\right) \dot{X}\right) a / 2=0 \\
& 8 \pi \mu \rho^{3} \dot{\Omega}_{Z}+\left(r N \lambda\left(C_{n}-C_{s}\right)\left(\omega_{2}-\omega_{3}\right) \sin \beta-F_{3}\right) a \sqrt{3} / 2=0
\end{aligned}
$$

From Equations (17)-(20), one can obtain the relations for $\dot{X}, \dot{\Omega}_{X}, \dot{\Omega}_{Y}, \dot{\Omega}_{Z}$ respectively. This result would be in the form of state space equations, and can be described as [14]

$$
\begin{gathered}
\dot{X}=C_{1}\left(\sum_{i=1}^{3} \omega_{i}\right) \\
\dot{\Omega}_{X}=C_{2}\left(\sum_{i=1}^{3} \omega_{i}\right) \\
\dot{\Omega}_{Y}=C_{3}\left(2 \omega_{1}-\omega_{2}-\omega_{3}\right) \\
\dot{\Omega}_{Z}=C_{4}\left(\omega_{3}-\omega_{2}\right)
\end{gathered}
$$

where the parameters $C_{1}$ through $C_{4}$ can be determined by the robot and fluid characteristic parameters. A more detailed derivation of these equations are mentioned in [14].

It should be noted that the microrobot kinematics has non-holonomic constraint [33]. The non-holonomic constraint and the underactuated property should be considered in designing the controller. Therefore, all of the 5-DoFs cannot be tracked simultaneously, and the position and orientation are controlled in turn. The system control is as follows.

\section{System Control}

To reach 5-DOF tracking control according to the four governing equations, it is pinpointed to control the roll and pitch angle without locational displacement. The input of the controlling system is angular velocity of the helixes. Orientation can be defined by vector of the robot relative to the reference frame that is

$$
\vec{X}=\left[\begin{array}{lll}
\cos \Omega_{y} \cos \Omega_{z} & \cos \Omega_{y} \sin \Omega_{z} & -\sin \Omega_{y}
\end{array}\right]^{T}
$$

The first derivative of Equation (25) is the nonholonomic constraint of underwater vehicles as mentioned above [33], and can be better understood by referring to Figure 1. Any desired approach vector, $\vec{X}_{d}$, is reachable by computation of suitable $\Omega_{y}$ and $\Omega_{z}$ through Equation (23), Equation (24) and initial conditions. Hence, there will remain two independent control inputs to achieve the desirable roll and pitch angles. In order to provide the no displacement condition, the third input has to satisfy the following condition:

$$
\sum_{i=1}^{3} \omega_{i}=0
$$

Therefore, in order to reach any desired location, firstly the robot vector needs to be aligned with the desired displacement vector by pure rotation of the robot and then propel to the desired location by equal maximum rotation of all of the flagella which provides translation in minimum time with constant approach vector concerning Equations (21)-(25). After the robot reaches the desired location, to reach the desired approach vector, the robot will purely rotate as before. 


\subsection{Error Definition}

To determine the error of angular velocity of each flagellum for the orientation control, the normalized directed distance of helix axes from the effective rotation axis, $\vec{X}_{d} \times \vec{X}$, are used as follows:

$$
E_{i}=\frac{\vec{p}_{i} \cdot\left(\left(\vec{X}_{d} \times \vec{X}\right) \times \vec{X}\right)}{a} \quad i=1,2,3
$$

and

$$
\begin{aligned}
& {\left[\overrightarrow{p_{1}}\left|\overrightarrow{p_{2}}\right| \overrightarrow{p_{3}}\right]=\left[\begin{array}{c}
\sin \Omega_{x} \sin \Omega_{z}+\cos \Omega_{x} \cos \Omega_{z} \sin \Omega_{y} \\
\cos \Omega_{x} \sin \Omega_{z} \sin \Omega_{y}-\cos \Omega_{z} \sin \Omega_{x} \\
\cos \Omega_{x} \sin \Omega_{y}
\end{array}\right.} \\
& \frac{\sqrt{3} \cos \Omega_{x} \sin \Omega_{z}}{2}-\frac{\sqrt{3} \cos \Omega_{z} \sin \Omega_{x} \sin \Omega_{y}}{2}-\frac{\sin \Omega_{x} \sin \Omega_{z}}{2}-\frac{\cos \Omega_{x} \cos \Omega_{z} \sin \Omega_{y}}{2} \\
& \frac{\cos \Omega_{z} \sin \Omega_{x}}{2}-\frac{\sqrt{3} \cos \Omega_{x} \cos \Omega_{z}}{2}-\frac{\sqrt{3} \sin \Omega_{x} \sin \Omega_{z} \sin \Omega_{y}}{2}-\frac{\cos \Omega_{x} \sin \Omega_{z} \sin \Omega_{y}}{2} \\
& -\frac{\cos \Omega_{x} \cos \Omega_{y}}{2}-\frac{\sqrt{3} \sin \Omega_{x} \cos \Omega_{y}}{2} \\
& \left.\begin{array}{c}
-\frac{\sqrt{3} \cos \Omega_{x} \sin \Omega_{z}}{2}+\frac{\sqrt{3} \cos \Omega_{z} \sin \Omega_{x} \sin \Omega_{y}}{2}-\frac{\sin \Omega_{x} \sin \Omega_{z}}{2}-\frac{\cos \Omega_{x} \cos \Omega_{z} \sin \Omega_{y}}{2} \\
\frac{\cos \Omega_{z} \sin \Omega_{x}}{2}+\frac{\sqrt{3} \cos \Omega_{x} \cos \Omega_{z}}{2}+\frac{\sqrt{3} \sin \Omega_{x} \sin \Omega_{z} \sin \Omega_{y}}{2}-\frac{\cos \Omega_{x} \sin \Omega_{z} \sin \Omega_{y}}{2} \\
-\frac{\cos \Omega_{x} \cos \Omega_{y}}{2}+\frac{\sqrt{3} \sin \Omega_{x} \cos \Omega_{y}}{2}
\end{array}\right]
\end{aligned}
$$

Therefore, the defined errors are established by the geometry of the system.

\subsection{Fuzzy-PI Controller}

The robustness and compatibility of controlling processes can be granted by fuzzy logic approach. However, any designed controller needs to be as simple as possible to avoid any unnecessary computation leading to inevitable errors. As far as control error of the system is of high precision, the easiest way to use fuzzy logic algorithms in a controlling process for providing robustness and compatibility is to combine it with traditional controllers.

The maximum magnitude of the angular velocity of the flagella is considered between $25 \mathrm{~Hz}$ to $50 \mathrm{~Hz}$ for gains specification, and $C_{1}$ through $C_{4}$ are considered as $0.0213,0.0227,0.0184$, and 0.0160 respectively [14].

P-controller can be used with the same gain for the three errors because $\overrightarrow{p_{1}}+\overrightarrow{p_{2}}+\overrightarrow{p_{3}}=0$. Therefore, $E_{1}+E_{2}+E_{3}=0$. Even though a simple P-controller has an acceptable rise time, the steady state error is inevitable. Because of the systems dynamical orientation, the PI controller is chosen as a suitable, adequate controller and to prevent unnecessary computational load when the angular distance between the desired and current approach vector is more than $6^{\circ}$ the P-controller will be on board. To adjust the proportional and integral coefficients of the PI controller performing within $0^{\circ}$ to $6^{\circ}$ angular distance, the fuzzy logic algorithm is used.

Concerning the method of error detection, in order to establish suitable fuzzy rule base at the time that the fuzzy controller is on board, it was decided to use $|E|$ and $d|E| / d t$ as the fuzzy logic algorithm inputs. So, the fuzzy rule base is formed according to $|E|$ and $d|E| / d t$ as shown in Tables 1 and 2.

In Tables 1 and 2, fuzzy rules should be written based on the membership functions defined for each variable. For example, for tuning the gain $K_{p}$, depending on member functions for $d|E| / d t$ and $|E|$, the ith rule is represented. It is well-known that fuzzy controller is based on the IF-THEN rule as R1: if $d|E| / d t$ is NB, and $|E|$ is PS, then $K_{p}$ is B; R2: if $d|E| / d t$ is NS, and $|E|$ is PM, then $K_{p}$ is S; R3: if $d|E| / d t$ is ZO, and $|E|$ is PB, then $K_{p}$ is SS; R4: if $d|E| / d t$ is PS, and $|E|$ is PS, then $K_{p}$ is S; R5: if $d|E| / d t$ is $\mathrm{PB}$, and $|E|$ is $\mathrm{ZO}$, then $K_{p}$ is $\mathrm{BB} \ldots$ 
Table 1. Fuzzy rules of $K_{p}$.

\begin{tabular}{cccccc}
\hline & & \multicolumn{4}{c}{$|\boldsymbol{E}|$} \\
\cline { 3 - 6 } & & ZO & PS & PM & PB \\
\hline \multirow{4}{*}{$d|E| / d t$} & NB & BB & B & B & M \\
& NM & BB & B & M & M \\
& NS & B & M & S & SS \\
& ZO & M & S & SS & SS \\
& PS & S & S & SS & SS \\
& PM & BB & SS & SS & SSS \\
& PB & BB & SS & SS & SSS \\
\hline
\end{tabular}

Table 2. Fuzzy rules of $K_{i}$.

\begin{tabular}{cccccc}
\hline & & \multicolumn{4}{c}{$|\boldsymbol{E}|$} \\
\cline { 3 - 6 } & & ZO & PS & PM & PB \\
\hline \multirow{4}{*}{$d|\boldsymbol{E}| / \boldsymbol{d} \boldsymbol{t}$} & NB & NM & NM & ZO & ZO \\
& NM & NM & NS & ZO & ZO \\
& NS & NS & ZO & PS & PS \\
& ZO & ZO & PS & PS & PM \\
& PS & PS & PS & PM & PM \\
& PM & PS & PM & PB & PB \\
& PB & PM & PB & PB & PB \\
\hline
\end{tabular}

The above rules are inspired from references [34], adjusted based on the nature of the error, and tuned based on the response of simulation. The intervals of $K_{P}$ and $K_{i}$ are selected mainly by trial and error as $[0,25]$, and $[-1,1]$ respectively.

For inputs and outputs of the fuzzy logic algorithm, triangular membership function uniformly distributed along each interval are used. Moreover, Mamdani's inference method is selected for the fuzzy implementation. To be precise, the implementation is modeled by Mamdani's product operator, $\mathrm{t}$-norm operator is Min, rule aggregation operator is Max, and because of the necessity of continuity, Centroid is chosen as the defuzzifier. The main schematic of the proposed fuzzy-PI controller is shown in Figure 2.

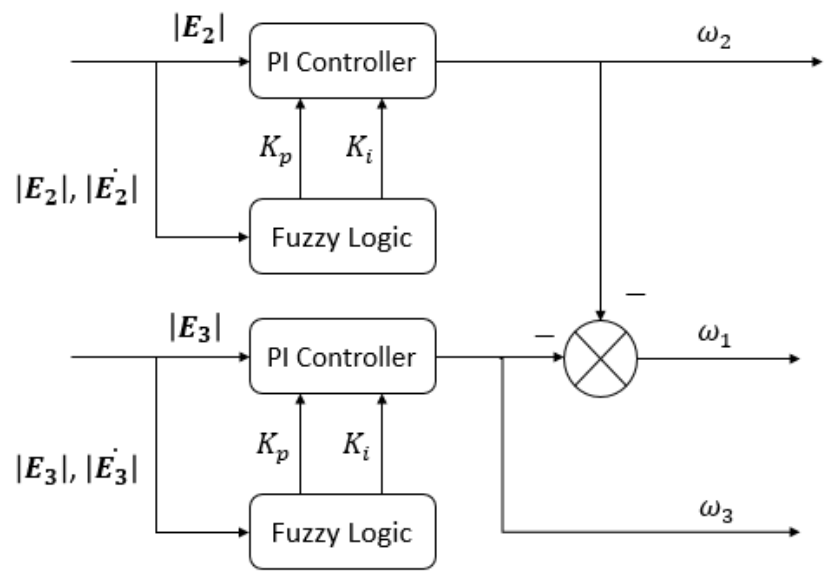

Figure 2. Control diagram of the fuzzy-PI controller.

As feasibility is the crucial aspect of any design, it has to be clarified. The matter for 3-DOF control of the robot is almost fully discussed in Ref. [14] with respect to the contemporary manufacturing technologies. The only critical aspect of 5-DOF manipulation of the underactuated microrobot is 
localization. As the localization of the robot has to have no conflict with the actuation system, among all of the sensory equipment which could provide the data of location and orientation, charge-coupled device array cameras in conjunction with microscopic lenses can be a promising solution [35]. All aside, the necessity of this study can be justified due to the novelty of microrobotic control area and due to the deficiency of proposed and controlled high-DOF micromanipulators as microrobots.

\section{Simulation Results}

Two fuzzy-PI controllers adjust PI parameters with two control inputs, and the third input adapts to satisfy the controlling condition of Equation (26).

As the main goal of the orientation controller is to maintain approach vector without locational displacement, and simple debugging of angular distances of the desired and current method vector of $0^{\circ}$ and $180^{\circ}$ were managed, three controllers including P-controller, fuzzy-PI controller without P-controller computational enhancement, and the proposed controller were tested for various desired orientations.

Now, let us discuss the effectivity of the defined error. Figure 3 shows an illustration of pure rotation control performance of the P-controller from the initial orientation $\left[\begin{array}{lll}1 & 0 & 0\end{array}\right]^{T}$ to the desired orientation $\left[-\frac{\sqrt{3}}{3} \frac{\sqrt{3}}{3} \frac{\sqrt{3}}{3}\right]^{T}$ which obtains acceptable rise time but also has steady state error as it is expected from a P-controller to have no deliberate effect on the steady state error. However, the P-controller utilizing the defined error can get near the target orientation, but it is troubled to handle the final dislocation.

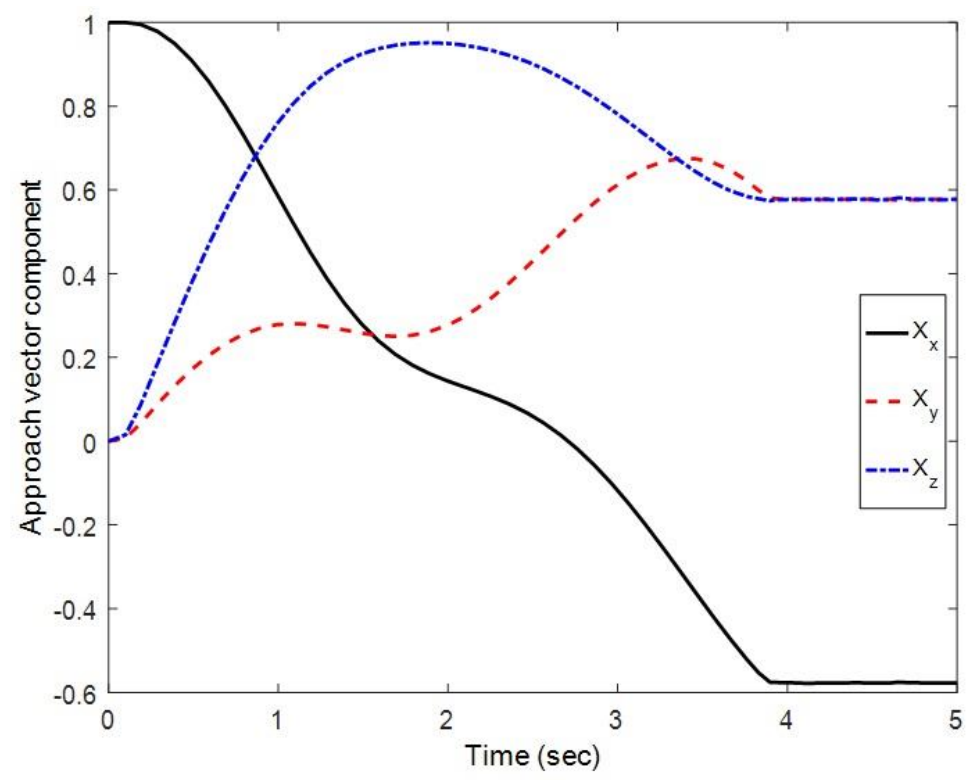

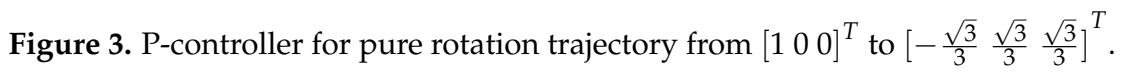

In scheming the design choices, a simple PI-controller seems to be sufficient to resolve the steady state error but a self-tuning PI-controller would always be a better choice as it can relatively resolve partial disturbances. Let us try the fuzzy-PI controller. Figure 4 depicts the pure rotation control performance of the fuzzy-PI controller without the P-controller computation enhancement from $\left[\begin{array}{lll}1 & 0 & 0\end{array}\right]^{T}$ to $\left[\begin{array}{lll}-1 & 0 & 0\end{array}\right]^{T}$ and its adaptive $K_{P}$, and $K_{i}$ as a function of time. The controller resolved the steady state error; however, the system was partially disturbed. 

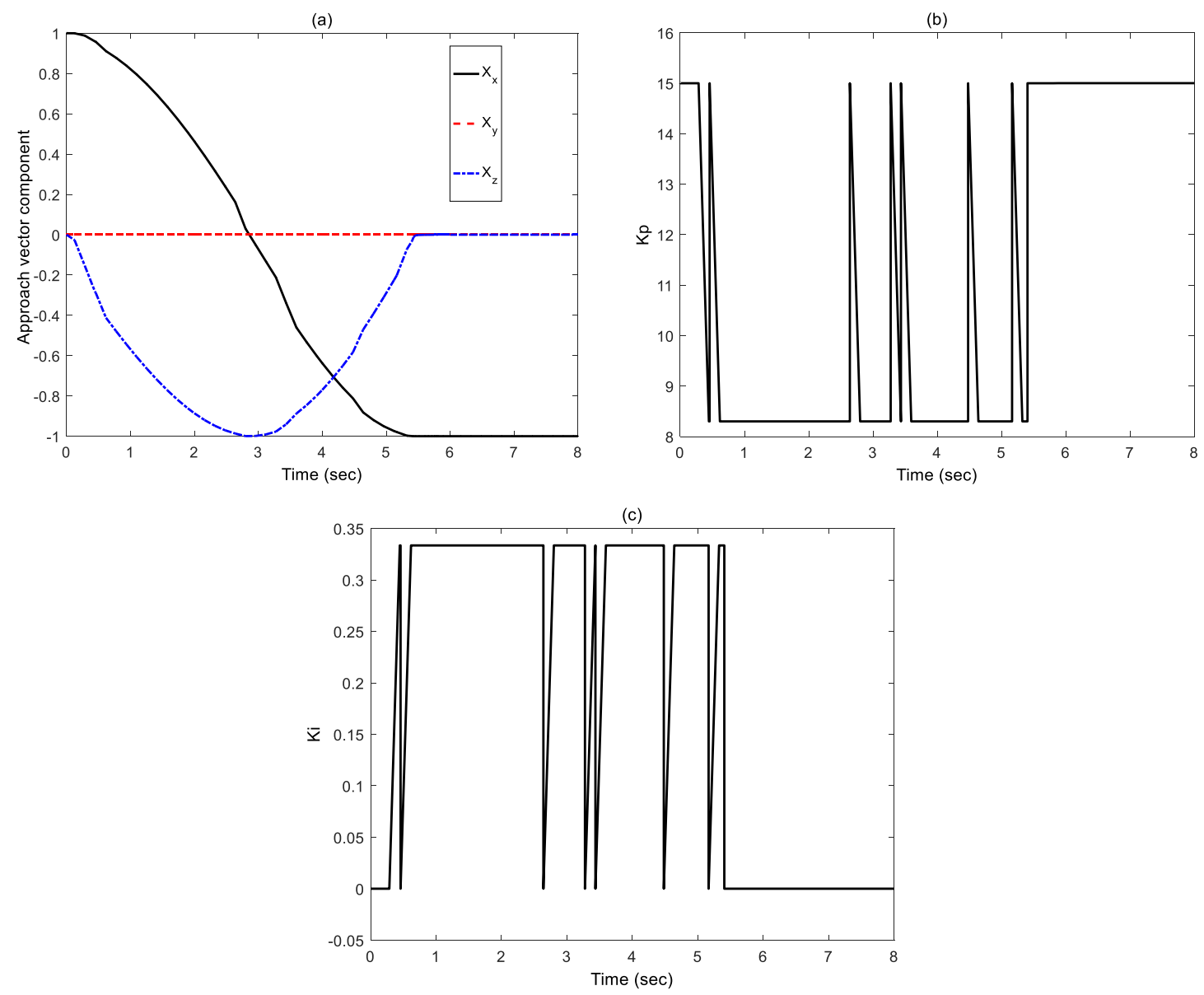

Figure 4. Fuzzy-PI controller without the P-controller enhancement: (a) pure rotation trajectory from the approach vector $\left[\begin{array}{lll}1 & 0 & 0\end{array}\right]^{T}$ to $\left[\begin{array}{lll}-1 & 0 & 0\end{array}\right]^{T} ;(\mathbf{b})$ variation of $K_{p} ;(\mathbf{c})$ variation of $K_{i}$

Figure 5 demonstrates pure rotation control of the proposed controller for two desired approach vectors which have good rise time and no steady state error. Comparing Figures $4 \mathrm{a}$ and $5 \mathrm{a}$, the proposed controller is swifter as it resolved the steady state error for the partial disturbed system. Figure $5 \mathrm{~b}$ illustrates the controller performance passing through one of the system's singularities.
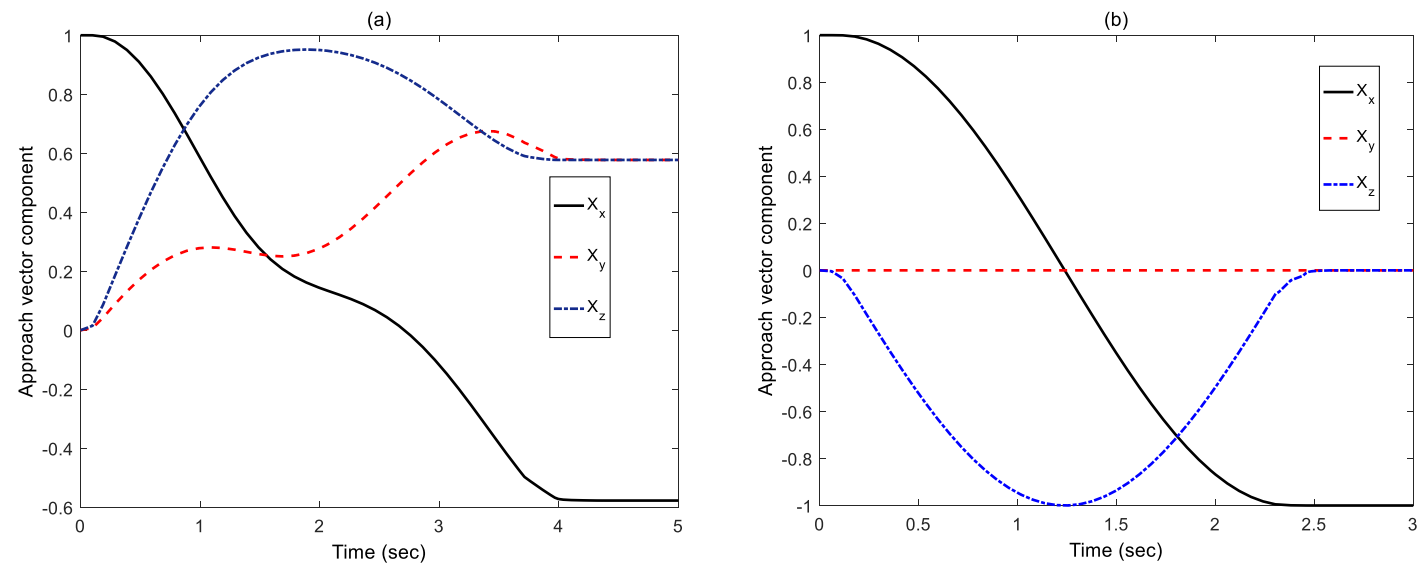

Figure 5. (a) Fuzzy-PI controller pure rotation trajectory from $\left[\begin{array}{lll}1 & 0 & 0\end{array}\right]^{T}$ to $\left[\begin{array}{lll}-\frac{\sqrt{3}}{3} & \frac{\sqrt{3}}{3} & \frac{\sqrt{3}}{3}\end{array}\right]^{T} ;(\mathbf{b})$ Fuzzy-PI controller pure rotation trajectory from $\left[\begin{array}{lll}1 & 0 & 0\end{array}\right]^{T}$ to $\left[\begin{array}{lll}-1 & 0 & 0\end{array}\right]^{T}$. 
For achieving 5-DOF target position, three stages were proposed. First, the robot has to reach close enough to locational displacement vector, then starts to proceed to the desired location by simply increasing the flagella angular velocities equally, and finally, when the robot reaches the desired location, the robot will purely rotate toward the desired orientation. Figure 6 exhibits the trajectory tracking performance of the proposed controller.
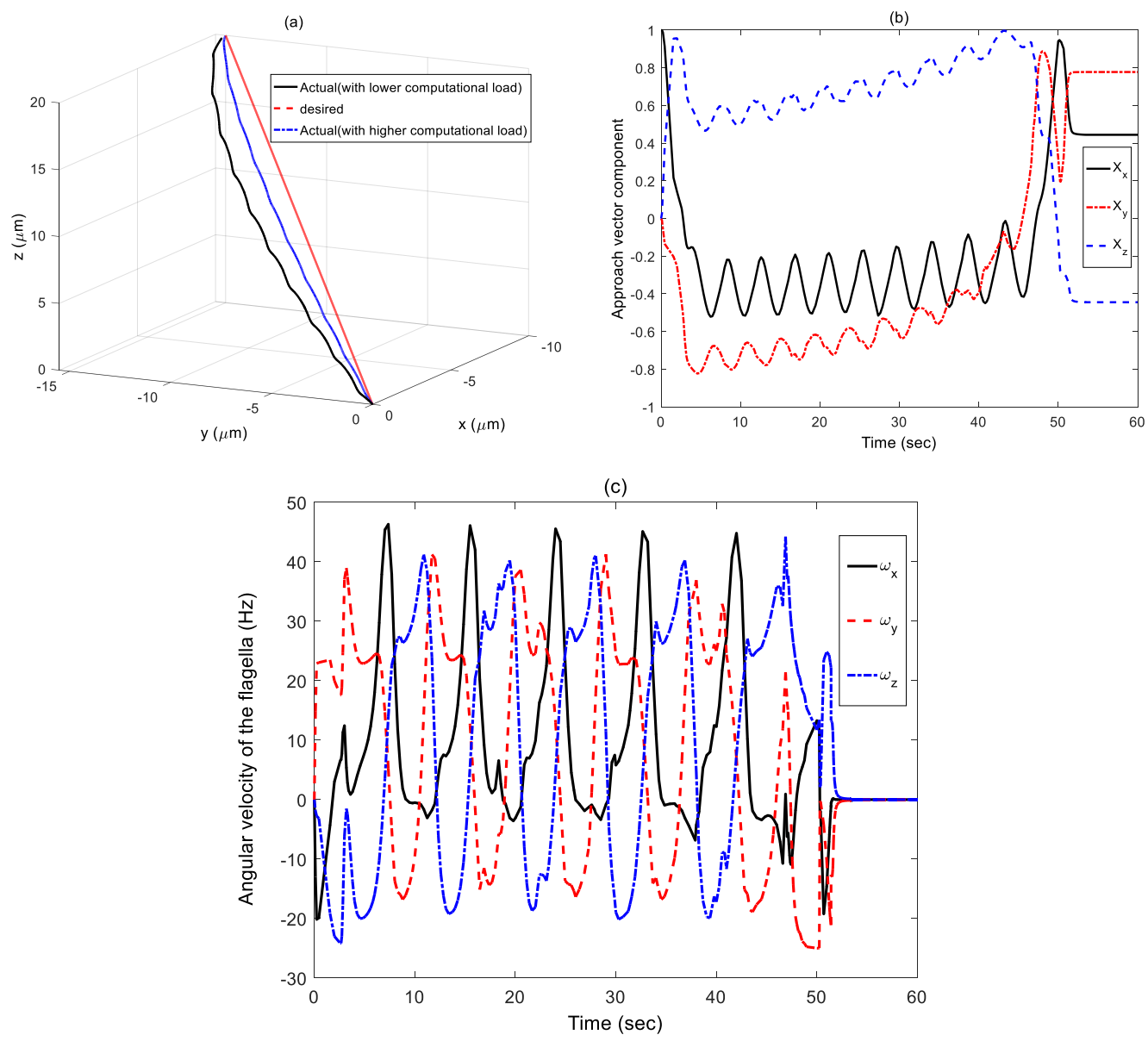

Figure 6. Trajectory tracking performance: (a) Location from $\left[\begin{array}{lll}0 & 0 & 0\end{array}\right]^{T}$ to $\left[\begin{array}{lll}-10 & -15 & 20\end{array}\right]^{T}$; (b) approach vector from $\left[\begin{array}{lll}1 & 0 & 0\end{array}\right]^{T}$ to $\left[\begin{array}{lll}\frac{4}{9} & \frac{7}{9} & \frac{-4}{9}\end{array}\right]^{T}$; and (c) overall helix angular velocities.

As Figure 6 shows, the controller performs the control performance correctly and thoroughly. The computational load is directly affected by the smallness of the boundary in which the approach vector and displacement vector are close enough to be considered "almost aligned" and the controller authorizes the propulsion. In Figure $6 \mathrm{~b}, \mathrm{c}$, almost after $50 \mathrm{~s}$ the location control is finished and pure rotation control begins.

The proposed system is based on three helixes as actuators. The other forms of actuations require specific dynamic modelling and controller design. The hydrodynamic drag is considered as the main fluid force. However, there are other forces to be considered such as electrostatic and Van der Waals forces.

\section{Conclusions}

An error detection method for multi-propulsion-unit systems is utilized for 5-DOF micromanipulation of an underactuated bio-inspired helical swimming microrobot by fuzzy-PI controller. The proposed controlling error definition extracted from the system geometry is general for similar actuation configurations. Increasing microrobots degrees of freedom only by proposing a 
suitable controller is one of the achievements of the present work. The proposed controller provides 5-DOF for the helical swimming microrobot in low Reynolds environments. With respect to the proposed controlling error, a simple p-controller was considered and tested but at last, for the sake of elimination of the steady state error and increment of robustness of the system a Fuzzy-PI controller was used to perform an effective trajectory regarding of systems governing equations.

Other than experimental validation of the proposed controller, future work for authors is to explore the use of sophisticated artificial intelligence ( $\mathrm{Al}$ ) methods (such as genetic programming, automated neural network search) for controlling movements of swimming microrobot. The advantages of $\mathrm{Al}$ method are the ability to build the explicit models for product quality as a function of system input process parameters based on only the data. This model can then be used offline or can be integrated in system for real-time monitoring of process.

Author Contributions: M.J.P. was responsible for simulation and implementing the control system to the dynamics. S.T. and R.V. participated in dynamic modelling and control respectively. M.M.A. helped with the fuzzy rules section and writing.

Funding: The research received no funding.

Conflicts of Interest: The authors declare no conflict of interest.

\section{References}

1. Khalil, I.S.; Magdanz, V.; Sanchez, S.; Schmidt, O.G.; Abelmann, L.; Misra, S. Magnetic control of potential microrobotic drug delivery systems: Nanoparticles, magnetotactic bacteria and self-propelled microjets. In Proceedings of the 35th Annual International Conference of the IEEE, Engineering in Medicine and Biology Society (EMBC), Osaka, Japan, 3-7 July 2013; pp. 5299-5302.

2. Devlin, P.M. Brachytherapy: Applications and Techniques; Springer Publishing Company: New York, NY, USA, 2015.

3. Trachoo, O.; Rivolta, M.N. Neural differentiation of human embryonic stem cells and their potential application in a therapy for sensorineural hearing loss. In Trends in Stem Cell Biology and Technology; Springer: New York, NY, USA, 2009; pp. 261-282.

4. Nelson, B.J.; Kaliakatsos, I.K.; Abbott, J.J. Microrobots for minimally invasive medicine. Annu. Rev. Biomed. Eng. 2010, 12, 55-85. [CrossRef] [PubMed]

5. Folio, D.; Ferreira, A. Two-dimensional robust magnetic resonance navigation of a ferromagnetic microrobot using pareto optimality. IEEE Trans. Robot. 2017, 33, 583-593. [CrossRef]

6. Kummer, M.P.; Abbott, J.J.; Kratochvil, B.E.; Borer, R.; Sengul, A.; Nelson, B.J. Octomag: An electromagnetic system for 5-dof wireless micromanipulation. IEEE Trans. Robot. 2010, 26, 1006-1017. [CrossRef]

7. Evans, A.A.; Lauga, E. Propulsion by passive filaments and active flagella near boundaries. Phys. Rev. E 2010, 82, 041915. [CrossRef] [PubMed]

8. Abbott, J.J.; Peyer, K.E.; Dong, L.X.; Nelson, B.J. How should microrobots swim. In Robotics Research; Springer: New York, NY, USA, 2010; pp. 157-167.

9. Floyd, S.; Pawashe, C.; Sitti, M. Microparticle manipulation using multiple untethered magnetic micro-robots on an electrostatic surface. In Proceedings of the IROS 2009. IEEE/RSJ International Conference on Intelligent Robots and Systems, St. Louis, MO, USA, 10-15 October 2009; pp. 528-533.

10. Dijkink, R.; Van Der Dennen, J.; Ohl, C.; Prosperetti, A. The 'acoustic scallop': A bubble-powered actuator. J. Micromech. Microeng. 2006, 16, 1653. [CrossRef]

11. Yang, L.; Wang, Q.; Vong, C.-I.; Zhang, L. A miniature flexible-link magnetic swimming robot with two vibration modes: Design, modeling and characterization. IEEE Robot. Autom. Lett. 2017, 2, 2024-2031. [CrossRef]

12. Huang, C.; Lv, J.-A.; Tian, X.; Wang, Y.; Yu, Y.; Liu, J. Miniaturized swimming soft robot with complex movement actuated and controlled by remote light signals. Sci. Rep. 2015, 5, 17414. [CrossRef] [PubMed]

13. Arcese, L.; Fruchard, M.; Ferreira, A. Endovascular magnetically guided robots: Navigation modeling and optimization. IEEE Trans. Biomed. Eng. 2012, 59, 977-987. [CrossRef] [PubMed] 
14. Nourmohammadi, H.; Keighobadi, J.; Bahrami, M. Design, dynamic modelling and control of a bio-inspired helical swimming microrobot with three-dimensional manoeuvring. Trans. Inst. Meas. Control. 2016, 39, 1037-1046. [CrossRef]

15. Tabak, A.F.; Yesilyurt, S. Experiment-based kinematic validation of numeric modeling and simulated control of an untethered biomimetic microrobot in channel. In Proceedings of the 12th IEEE International Workshop on Advanced Motion Control (AMC), Sarajevo, Bosnia-Herzegovina, 25-27 March 2012; pp. 1-6.

16. Flynn, A.M.; Tavrow, L.S.; Bart, S.F.; Brooks, R.A.; Ehrlich, D.J.; Udayakumar, K.R.; Cross, L.E. Piezoelectric micromotors for microrobots. J. Microelectromech. Syst. 1992, 1, 44-51. [CrossRef]

17. Tadokoro, S.; Murakami, T.; Fuji, S.; Kanno, R.; Hattori, M.; Takamori, T.; Oguro, K. An elliptic friction drive element using an icpf actuator. IEEE Control. Syst. 1997, 17, 60-68. [CrossRef]

18. Magdanz, V.; Sanchez, S.; Schmidt, O.G. Development of a sperm-flagella driven micro-bio-robot. Adv. Mater. 2013, 25, 6581-6588. [CrossRef] [PubMed]

19. Belharet, K.; Folio, D.; Ferreira, A. Three-dimensional controlled motion of a microrobot using magnetic gradients. Adv. Robot. 2011, 25, 1069-1083. [CrossRef]

20. Marino, H.; Bergeles, C.; Nelson, B.J. Robust electromagnetic control of microrobots under force and localization uncertainties. IEEE Trans. Autom. Sci. Eng. 2014, 11, 310-316. [CrossRef]

21. Arcese, L.; Fruchard, M.; Ferreira, A. Adaptive controller and observer for a magnetic microrobot. IEEE Trans. Robot. 2013, 29, 1060-1067. [CrossRef]

22. Rovetta, A.; Wen, X. Fuzzy logic in robot grasping control. In Proceedings of the IROS'91, IEEE/RSJ International Workshop on Intelligent Robots and Systems '91: Intelligence for Mechanical Systems, Osaka, Japan, 3-5 November 1991; pp. 1632-1637.

23. Katbab, A. Fuzzy logic and controller design-a review. In Proceedings of the Southeastcon'95: Visualize the Future, Raleigh, NC, USA, 26-29 March 1995; pp. 443-449.

24. Yoo, B.K.; Ham, W.C. Adaptive control of robot manipulator using fuzzy compensator. IEEE Trans. Fuzzy Syst. 2000, 8, 186-199.

25. Al-Hadithi, B.M.; Matia, F.; Jimenez, A. Robust fuzzy control for a mobile robot. In Proceedings of the World, Automation Congress (WAC 2008), Hawaii, HI, USA, 28 September-2 October 2008; pp. 1-6.

26. Paliwal, S.; Chopra, V.; Singla, S.K. Stabilization of mobile inverted pendulum using fuzzy pid controllers. In Proceedings of the 7th India International Conference on Power Electronics (IICPE), Patiala, India, 17-19 November 2016; pp. 1-4.

27. Li, H.; Huang, Y.; Lu, J. Reactive power compensation and dc link voltage control using fuzzy-pi on grid-connected pv system with d-statcom. In Proceedings of the Power and Energy Engineering Conference (APPEEC), 2016 IEEE PES Asia-Pacific, Xi'an, China, 25-28 October 2016; pp. 1240-1244.

28. Korba, P.; Babuska, R.; Verbruggen, H.B.; Frank, P.M. Fuzzy gain scheduling: Controller and observer design based on Lyapunov method and convex optimization. IEEE Trans. Fuzzy Syst. 2003, 11, 285-298. [CrossRef]

29. Palm, R.; Driankov, D. Design of a fuzzy gain scheduler using sliding mode control principles. Fuzzy Sets Syst. 2001, 121, 13-23. [CrossRef]

30. Nguyen, K.-D.; Dankowicz, H. Adaptive control of underactuated robots with unmodeled dynamics. Robot. Auton. Syst. 2015, 64, 84-99. [CrossRef]

31. Korkmaz, S. A review of active structural control: Challenges for engineering informatics. Comput. Struct. 2011, 89, 2113-2132. [CrossRef]

32. Chwang, A.; Wu, T.Y. A note on the helical movement of micro-organisms. Proc. R. Soc. Lond. B Biol. Sci. 1971, 178, 327-346. [CrossRef] [PubMed]

33. Yuan, H. Control of Nonholonomic Systems; University of Central Florida: Orlando, FL, USA, 2009.

34. De Carli, A.; Liguori, P.; Marroni, A. A fuzzy-pi control strategy. Control. Eng. Pract. 1994, 2, 147-153. [CrossRef]

35. Ullrich, F.; Bergeles, C.; Pokki, J.; Ergeneman, O.; Erni, S.; Chatzipirpiridis, G.; Pané, S.; Framme, C.; Nelson, B.J. Mobility experiments with microrobots for minimally invasive intraocular surgerymicrorobot experiments for intraocular surgery. Investig. Ophthalmol. Vis. Sci. 2013, 54, 2853-2863. [CrossRef] [PubMed]

(C) 2018 by the authors. Licensee MDPI, Basel, Switzerland. This article is an open access article distributed under the terms and conditions of the Creative Commons Attribution (CC BY) license (http://creativecommons.org/licenses/by/4.0/). 